\title{
Four-zero neutrino Yukawa textures in the minimal seesaw framework
}

\author{
Gustavo C. Branco, ${ }^{1, *}$ David Emmanuel-Costa, ${ }^{1, \dagger}$ M. N. Rebelo, ${ }^{1,2, \ddagger}$ and Probir Roy ${ }^{3,4, \S}$ \\ ${ }^{1}$ Departamento de Física and Centro de Física Teórica de Partículas (CFTP), Instituto Superior Técnico (IST), Avenida Rovisco Pais, \\ 1049-001 Lisboa, Portugal \\ ${ }^{2}$ CERN, Department of Physics, Theory Division, CH-1211 Geneva 23, Switzerland \\ ${ }^{3}$ Tata Institute of Fundamental Research, Homi Bhabha Road, Mumbai 400 005, India \\ ${ }^{4}$ Saha Institute of Nuclear Physics, Block AF, Sector 1, Kolkata 700 064, India
}

(Received 14 December 2007; published 25 March 2008)

\begin{abstract}
We investigate, within the type I seesaw framework, the physical implications of zero textures in the Yukawa couplings which generate the neutrino Dirac mass matrix $m_{D}$. It is shown that four is the maximal number of texture zeros compatible with the observed leptonic mixing and the assumption that no neutrino mass vanishes. We classify all allowed four-zero textures of $m_{D}$ into two categories with three classes each. We show that the different classes, in general, admit $C P$ violation both at low and high energies. We further present the constraints obtained for low energy physics in each case. The role of these zero textures in establishing a connection between leptogenesis and low energy data is analyzed in detail. It is shown that it is possible in all cases to completely specify the parameters relevant for leptogenesis in terms of light neutrino masses and leptonic mixing together with the unknown heavy neutrino masses.
\end{abstract}

DOI: 10.1103/PhysRevD.77.053011

PACS numbers: 14.60.Pq, 11.30.Er

\section{INTRODUCTION}

Recent impressive experimental progress towards determining the masses and mixing angles of the three known light neutrinos has brought urgency to the task of unraveling the flavor structure of the neutrino mass matrix $m_{\nu}$. It has been pointed out [1] that, in the case of Majorana neutrinos, it is not possible to completely determine the structure of $m_{\nu}$ from feasible experiments. This is one of the motivations for introducing some theoretical input aimed at reducing the number of free parameters. One interesting possibility is the imposition of zeros in the elements of $m_{\nu}$ [1]. Another is to assume the vanishing of $\operatorname{det} m_{\nu}$ [2]. Several papers have analyzed in detail the consequences of imposing zeros directly in the elements of $m_{\nu}$, starting with at least two-zero textures [1,3]. Implications of single texture zeros were studied in detail in [4]. In fact, the existence of vanishing mass matrix elements may reflect the presence of a family symmetry acting in the leptonic sector [5,6]. It is then more natural to investigate and classify the appearance of zeros in the fundamental mass matrix appearing in the Lagrangian rather than in $m_{\nu}$ which, at least within the seesaw framework, is a derived quantity. Therefore we focus our attention on the Yukawa couplings which lead to the neutrino Dirac mass matrix $m_{D}$, once spontaneous symmetry breaking occurs. One can then see how zeros in $m_{D}$ affect $m_{\nu}$ which we take to be related to $m_{D}$ by the type I seesaw relation. Throughout, by "texture" we shall refer to a

\footnotetext{
*gbranco@ist.utl.pt

†david.costa@ist.utl.pt

Presently at CERN on sabbatical leave from IST.

margarida.rebelo@cern.ch; rebelo@ist.utl.pt

${ }^{\S}$ Presently at SINP.

probirr@gmail.com
}

configuration of $m_{D}$ containing zeros in some of its elements. In the Froggatt-Nielsen approach [7] texture zeros correspond to extremely suppressed entries, which can be taken effectively as zeros. The stability of zeros in neutrino mass matrices under quantum corrections in type I seesaw models has been studied in Refs. [8-10]. One also needs to be aware that renormalization group effects can be quite large in the case of quasidegenerate (inverted hierarchical) light neutrino masses $[11,12]$.

In this paper we classify and analyze the physical implications of all neutrino Yukawa coupling matrices with four-zero textures in the weak basis (WB), where the charged lepton and the right-handed Majorana neutrino mass matrices are diagonal and real. For simplicity, we work within the framework of the type I seesaw mechanism, where three right-handed singlet neutrinos are added to the standard model (SM). The case of only two righthanded heavy neutrinos leads to one zero neutrino mass, and in this case only one-zero textures and some of the two-zero textures are allowed experimentally $[13,14]$. With three heavy right-handed neutrinos and the additional requirement that none of the physical neutrino masses vanish, we show that four is the maximal number of zeros in textures of $m_{D}$ that are compatible with the available data on neutrino mixing. We organize all such four-zero textures into classes and discuss the physical implications of each class. The imposition of texture zeros in the Yukawa couplings has the advantage of allowing for the possibility of establishing a connection between low energy physics and physics at high energies, in particular, leptogenesis [15].

It is by now established that new sources of $C P$ violation beyond the Kobayashi-Maskawa mechanism of the SM are required in order to dynamically generate the observed baryon asymmetry of the Universe (BAU) [16-21]. The 
scenario of baryogenesis through leptogenesis has been rendered especially appealing by the discovery of neutrino oscillations, which provides evidence for nonvanishing neutrino masses. In general, there is no direct connection between $C P$ violation at low energies and that entering in leptogenesis [22,23]. It has been shown, however, that such a connection arises in models with texture zeros in $m_{D}$ $[1,13,24,25]$. This is a question that we analyze in the present work for each one of the classes of allowed fourzero textures, and we conclude that it is possible in all cases to completely specify the parameters relevant for leptogenesis in terms of light neutrino masses and leptonic mixing together with the unknown heavy neutrino masses.

Texture zeros are clearly not WB invariant. For definiteness we analyze the allowed four-zero textures in the WB in which both the charged lepton mass matrix and the heavy right-handed Majorana neutrino mass matrix are diagonal, as mentioned earlier. The question of how to recognize a flavor model corresponding to a given set of texture zeros, when written in a different basis, was addressed in Ref. [26]. It was shown there that some sets of texture zeros imply the vanishing of certain $C P$-odd WB invariants. The relevance of $C P$-odd $\mathrm{WB}$ invariants in the analysis of texture zero ansätze is due to the fact that texture zeros lead, in general, to a decrease in the number of $C P$-violating phases.

This paper is organized as follows. In Sec. II we set the notation and present our framework. We show in Sec. III, based on what is already known experimentally about leptonic mixing and on the condition that no neutrino mass vanishes, why textures with five or more zeros in $m_{D}$ are ruled out. In Sec. IV we enumerate the possible classes of four-zero textures that are allowed and give for each one of them low energy relations leading to physical constraints. $C P$ violation and related WB invariants for the surviving four-zero textures are discussed in Sec. V. In Sec. VI we analyze the physical implications of four-zero textures both for low energies and for leptogenesis. Our conclusions are summarized in Sec. VII.

\section{NOTATION AND FRAMEWORK}

We work in the context of the minimal type I seesaw mechanism with three generations of right-handed neutrinos which are singlets of $\mathrm{SU}(2)$. We do not extend the Higgs sector, and therefore we do not include Majorana mass terms for left-handed neutrinos. After spontaneous symmetry breaking, the leptonic mass terms are given by

$$
\begin{aligned}
\mathcal{L}_{m} & =-\left[\bar{\nu}_{L}^{0} m_{D} \nu_{R}^{0}+\frac{1}{2} \nu_{R}^{0 T} C M_{R} \nu_{R}^{0}+\bar{l}_{L}^{0} m_{l} l_{R}^{0}\right]+\text { H.c. } \\
& =-\left[\frac{1}{2} n_{L}^{T} C \mathcal{M}^{*} n_{L}+\bar{l}_{L}^{0} m_{l} l_{R}^{0}\right]+\text { H.c., }
\end{aligned}
$$

where $n_{L}$ is a column vector with $n_{L}^{T}=\left(\nu_{L}^{0},\left(\nu_{R}^{0}\right)^{c}\right)$, while $M_{R}, m_{D}$, and $m_{l}$, respectively, denote the right-handed neutrino Majorana mass matrix, the neutrino Dirac mass matrix, and the charged lepton mass matrix in family space. The superscript 0 signifies the fact that the corresponding fields are eigenstates of flavor. The matrix $\mathcal{M}$ is given by

$$
\mathcal{M}=\left(\begin{array}{cc}
0 & m_{D} \\
m_{D}^{T} & M_{R}
\end{array}\right)
$$

We assume that the scale of $M_{R}$ is much higher than the electroweak scale $v \simeq 246 \mathrm{GeV}$. Upon diagonalization of the matrix $\mathcal{M}$, we are left with $\mathcal{D}=\operatorname{diag}\left(m_{1}, m_{2}\right.$, $\left.m_{3}, M_{1}, M_{2}, M_{3}\right)$, containing three light and three heavy Majorana neutrinos. The charged current interactions can then be written as

$$
\mathcal{L}_{W}=-\frac{g}{\sqrt{2}}\left(\bar{l}_{i L} \gamma_{\mu} K_{i j} \nu_{j L}+\bar{l}_{i L} \gamma_{\mu} G_{i j} N_{j L}\right) W^{\mu}+\text { H.c. }
$$

where $\nu_{j}$ and $N_{j}$ denote the light and the heavy neutrinos, respectively, and $K$ and $G$ are $3 \times 3$ blocks of a unitary $6 \times$ 6 matrix that diagonalizes the symmetric matrix $\mathcal{M}$. The light neutrino masses and mixing angles are obtained to an excellent approximation from

$$
U^{\dagger} m_{\mathrm{eff}} U^{\star}=d,
$$

where $m_{\text {eff }}=-m_{D} M_{R}^{-1} m_{D}^{T} \equiv m_{\nu}$ computed in the WB where $m_{l}$ is diagonal, and $d=\operatorname{diag}\left(m_{1}, m_{2}, m_{3}\right)$. The unitary matrix $U$ in Eq. (4) is the Pontecorvo, Maki, Nakagawa, and Sakata (PMNS) matrix [27] relating the mass eigenstate neutrinos $\nu_{i}(i=1,2,3)$ to the flavor eigenstate neutrinos $\nu_{f}(f=e, \mu, \tau)$ by $\nu_{f L}=U_{f i} \nu_{i L}$. It coincides with $K$ in Eq. (3) up to corrections of order $v^{2} / M^{2}$, which we ignore. The Yukawa textures that we analyze are imposed in the weak basis where $M_{R}$ and $m_{l}$ are real and diagonal. In this $\mathrm{WB}$, all $C P$-violating phases are contained in $m_{D}$. From Eq. (7) and the definition of $m_{\text {eff }}$, one can write $m_{D}$ in the Casas and Ibarra parametrization [28]:

$$
m_{D}=i U \sqrt{d} R \sqrt{D},
$$

where $D$ stands for $M_{R}$ in the WB where it is diagonal and $R$ is a general complex orthogonal matrix. It is clear, by construction, that mixing and $C P$ violation at low energies are blind to the matrix $R$. However this last matrix is relevant for leptogenesis.

\section{INADMISSIBILITY OF MORE THAN FOUR-ZERO TEXTURES}

We shall now demonstrate that, in the framework specified earlier, all five-zero textures in $m_{D}$ are ruled out. Let us start with the general form

$$
m_{D}=\left[\begin{array}{lll}
a_{1} & a_{2} & a_{3} \\
b_{1} & b_{2} & b_{3} \\
c_{1} & c_{2} & c_{3}
\end{array}\right] .
$$

Since we are assuming that none of the neutrino masses 
vanishes, we conclude from the definition of $m_{\text {eff }}$ that the determinant of $m_{D}$ cannot be zero. Therefore patterns of $m_{D}$ with one full row of zeros or one full column of zeros are ruled out, as well as patterns with zeros distributed in a quartet, i.e. in four elements (ij), $(l \mathrm{k}),(\mathrm{ik}),(l \mathrm{j})$, where $\mathrm{i}, \mathrm{j}, \mathrm{k}$, $l$ can be 1,2 or 3 , with $l \neq \mathrm{i}, \mathrm{k} \neq \mathrm{j}$. We are thus left with patterns where the zeros are placed in such a way that invariably two rows and two columns would have two zeros simultaneously. Together with the requirement that there is no quartet of zeros, this leads to several different possibilities where, in each case, one nonzero entry of $m_{D}$ is in a row and a column where all other entries are zeros. Some examples are

$$
\begin{array}{lll}
{\left[\begin{array}{ccc}
a_{1} & a_{2} & 0 \\
0 & 0 & b_{3} \\
c_{1} & 0 & 0
\end{array}\right],} & {\left[\begin{array}{ccc}
a_{1} & a_{2} & 0 \\
0 & 0 & b_{3} \\
0 & c_{2} & 0
\end{array}\right],} \\
{\left[\begin{array}{ccc}
0 & a_{2} & 0 \\
0 & b_{2} & b_{3} \\
c_{1} & 0 & 0
\end{array}\right],} & {\left[\begin{array}{ccc}
a_{1} & 0 & 0 \\
0 & b_{2} & 0 \\
0 & c_{2} & c_{3}
\end{array}\right],} \\
{\left[\begin{array}{ccc}
0 & a_{2} & a_{3} \\
b_{1} & 0 & 0 \\
0 & c_{2} & 0
\end{array}\right],} & {\left[\begin{array}{ccc}
0 & a_{2} & a_{3} \\
0 & b_{2} & 0 \\
c_{1} & 0 & 0
\end{array}\right] .}
\end{array}
$$

Since we work in a WB where $M_{R}$ is diagonal, the resulting matrix $m_{\text {eff }}$, for the five-zero textures under discussion, is always block diagonal. Furthermore, the fact that we are in a WB where the charged lepton mass matrix is also diagonal implies that these textures lead to twofamily mixing only, which is already ruled out experimentally. Indeed, it is already known that there are two large mixing angles in the PMNS matrix, and as a result, all fivezero textures are ruled out.

\section{FOUR-ZERO TEXTURES}

In this section, we classify all different possible fourzero textures for $m_{D}$ in a WB, where $M_{R}$ and $m_{l}$ are real and diagonal with no vanishing neutrino mass. Among patterns of four-zero textures in $m_{D}$, the nonvanishing det $m_{D}$ condition rules out the occurrence of three of the zeros in the same row or in the same column, as well as zeros distributed in a quartet, as explained in the previous section. Block diagonal patterns such as

$$
\begin{gathered}
{\left[\begin{array}{ccc}
a_{1} & 0 & 0 \\
0 & b_{2} & b_{3} \\
0 & c_{2} & c_{3}
\end{array}\right], \quad\left[\begin{array}{ccc}
a_{1} & 0 & a_{3} \\
0 & b_{2} & 0 \\
c_{1} & 0 & c_{3}
\end{array}\right],} \\
{\left[\begin{array}{ccc}
a_{1} & a_{2} & 0 \\
b_{1} & b_{2} & 0 \\
0 & 0 & c_{3}
\end{array}\right]}
\end{gathered}
$$

lead to two-family mixing only and are therefore ruled out.

The allowed remaining patterns can be split into two categories:

(i) those with two orthogonal rows; (ii) those with two orthogonal columns and no pairs of orthogonal rows.

The first category can be divided into three classes corresponding to

(i)(a) orthogonality of the first and second rows, leading to

$$
m_{\mathrm{eff} 12}=m_{\mathrm{eff} 21}=0
$$

(i)(b) orthogonality of the first and third rows, leading to

$$
m_{\mathrm{eff} 13}=m_{\mathrm{eff} 31}=0 ;
$$

(i)(c) orthogonality of the second and third rows, leading to

$$
m_{\mathrm{eff} 23}=m_{\mathrm{eff} 32}=0 .
$$

There are 18 different cases in (i)(a). Six of them have two zeros in the first row and two zeros in the second row, as, for example,

$$
\left[\begin{array}{ccc}
0 & 0 & a_{3} \\
0 & b_{2} & 0 \\
c_{1} & c_{2} & c_{3}
\end{array}\right], \quad\left[\begin{array}{ccc}
0 & 0 & a_{3} \\
b_{1} & 0 & 0 \\
c_{1} & c_{2} & c_{3}
\end{array}\right] .
$$

Another six different cases have two zeros in the first row, one zero in the second row, and one zero in the third row, as in

$$
\left[\begin{array}{ccc}
0 & a_{2} & 0 \\
b_{1} & 0 & b_{3} \\
0 & c_{2} & c_{3}
\end{array}\right], \quad\left[\begin{array}{ccc}
0 & a_{2} & 0 \\
b_{1} & 0 & b_{3} \\
c_{1} & c_{2} & 0
\end{array}\right] .
$$

Finally, six different cases are obtained with one zero in the first row, two zeros in the second row, and one zero in the third row, as in

$$
\left[\begin{array}{ccc}
0 & a_{2} & a_{3} \\
b_{1} & 0 & 0 \\
c_{1} & 0 & c_{3}
\end{array}\right], \quad\left[\begin{array}{ccc}
0 & a_{2} & a_{3} \\
b_{1} & 0 & 0 \\
c_{1} & c_{2} & 0
\end{array}\right]
$$

There are another 18 different cases in (i)(b). These are obtained from those in (i)(a), exchanging the second with the third row. The cases in (i)(c) are also 18 different ones obtained from those in (i)(a) by exchanging the first row with the third one. Each case in category (i) has one symmetric pair of nondiagonal zero entries in $m_{\text {eff }}$. Since $m_{\text {eff }}$ is symmetric by construction, due to its Majorana character, off-diagonal zeros always come in pairs.

Textures in category (ii) are obtained by transposing those in category (i) and discarding those already considered in (i). There are 18 cases altogether in category (ii). In all of these, two columns are orthogonal to each other, each having two zeros, and there is one column without zeros. This category can again be divided into three classes: 
(ii)(a) six cases with two zeros in the first row; these cases are given explicitly by

$$
\begin{array}{ll}
{\left[\begin{array}{lcc}
0 & 0 & a_{3} \\
0 & b_{2} & b_{3} \\
c_{1} & 0 & c_{3}
\end{array}\right],} & {\left[\begin{array}{ccc}
0 & 0 & a_{3} \\
b_{1} & 0 & b_{3} \\
0 & c_{2} & c_{3}
\end{array}\right],} \\
{\left[\begin{array}{lcc}
0 & a_{2} & 0 \\
0 & b_{2} & b_{3} \\
c_{1} & c_{2} & 0
\end{array}\right],} & {\left[\begin{array}{ccc}
0 & a_{2} & 0 \\
b_{1} & b_{2} & 0 \\
0 & c_{2} & c_{3}
\end{array}\right],} \\
{\left[\begin{array}{ccc}
a_{1} & 0 & 0 \\
b_{1} & b_{2} & 0 \\
c_{1} & 0 & c_{3}
\end{array}\right],} & {\left[\begin{array}{ccc}
a_{1} & 0 & 0 \\
b_{1} & 0 & b_{3} \\
c_{1} & c_{2} & 0
\end{array}\right] .}
\end{array}
$$

These verify the conditions

$$
\begin{gathered}
\left|m_{\mathrm{eff} 11} m_{\mathrm{eff} 23}\right|=\left|m_{\mathrm{eff} 12} m_{\mathrm{eff} 13}\right|, \\
\arg \left(m_{11} m_{23} m_{12}^{*} m_{13}^{*}\right)=0 .
\end{gathered}
$$

Note that $\arg \left(m_{i i} m_{j k} m_{i j}^{*} m_{i k}^{*}\right)$ is rephasing invariant. In Ref. [26] one example of this class was discussed.

(ii)(b) six cases with two zeros in the second row, which are obtained from the patterns in (ii)(a) by interchanging the first with the second row. These verify the conditions

$$
\begin{gathered}
\left|m_{\mathrm{eff} 22} m_{\mathrm{eff} 13}\right|=\left|m_{\mathrm{eff} 21} m_{\mathrm{eff} 23}\right|, \\
\arg \left(m_{22} m_{13} m_{21}^{*} m_{23}^{*}\right)=0 .
\end{gathered}
$$

(ii)(c) six cases with two zeros in the third row, which are obtained from the patterns in (ii)(a) by interchanging the first row with the third row. These verify the conditions

$$
\begin{gathered}
\left|m_{\mathrm{eff33}} m_{\mathrm{eff} 12}\right|=\left|m_{\mathrm{eff3} 31} m_{\mathrm{eff32}}\right|, \\
\arg \left(m_{33} m_{12} m_{31}^{*} m_{32}^{*}\right)=0 .
\end{gathered}
$$

Equations (16)-(18) are of the form

$$
m_{\text {eff } i i} m_{\text {eff } j k}=m_{\text {eff } i j} m_{\text {eff } i k}
$$

with $i, j, k$ different from each other and no sum implied.

It can be checked that all allowed cases in category (i) as well as in category (ii) contain the same number of independent parameters in $m_{D}$, and in all such cases one can rephase away three of the phases. The counting of independent parameters in $m_{\text {eff }}$ is also the same in all cases, as will be seen in the next section. Moreover, we shall analyze in Sec. VI the implications of Eqs. (9)-(11) and (16)-(18) corresponding to the two categories, each one with three different classes.

Notice that, although we are considering weak bases with the maximum number of zeros allowed by experiment, together with the assumption that no neutrino mass vanishes, the resulting matrix $m_{\text {eff }}$ contains at most onezero nondiagonal entry. We are not considering here the possibility of fine-tuning between the parameters of $m_{D}$ and those of $M_{R}$ leading to additional zeros due to special cancellations. This indicates that imposing texture zeros in the WB where $m_{l}$ and $M_{R}$ are diagonal does not allow one to generate any of the two-zero patterns considered in Ref. [1]. It is already known that not all of these patterns can be realized through the seesaw mechanism $[29,30]$.

\section{V. $C P$ VIOLATION AND WEAK BASIS INVARIANTS}

We start by recalling the general counting of the number of parameters contained in the lepton mass matrices and then consider the special case of textures with four zeros in $m_{D}$. In the WB where $M_{R}$ and $m_{l}$ are diagonal and real, leptonic mixing and $C P$ violation are encoded in $m_{D}$, which is an arbitrary complex $3 \times 3$ matrix. The latter contains nine real moduli and nine phases. Of these, only six phases are physical, since three phases can be removed by simultaneous rephasing of $\nu_{L}, l_{L}$. So $m_{D}$ is left with nine real moduli plus six phases. Taking into account the three eigenvalues of $M_{R}$, we have in this WB a total of 18 parameters including six phases. This equals the number of physical parameters, to wit, three light neutrino masses, three heavy neutrino masses, plus six mixing angles and six $C P$-violating phases in the first three rows of a $6 \times 6$ complex unitary matrix [31] which we have denoted as $K$ and $G$ in Eq. (3). It is interesting to notice that the number of independent physical phases in Eq. (5) is also six, three in the PMNS matrix and three required to parametrize the orthogonal complex matrix $R$.

Textures with four zeros in $m_{D}$ lead to a strong reduction in the number of parameters, since there are only five real parameters and two phases after rephasing. This gives rise to interesting phenomenological implications which are analyzed in detail in the next section. In particular, it will be shown that, in all four-zero textures classified by us, the matrix $R$, which plays an important role in leptogenesis, can be fully expressed in terms of low energy parameters entering in $U$ and $d$. This establishes a direct connection between leptogenesis and low energy data. Moreover, this link exists both in the cases of unflavored and flavored leptogenesis.

In scenarios where flavor does not play an important role, the lepton number asymmetry resulting from the decay of the $N_{j}$ heavy Majorana neutrino is given by [32]

$$
\begin{aligned}
\varepsilon_{N_{j}}= & \frac{g^{2}}{M_{W}^{2}} \sum_{k \neq j}\left[\operatorname{Im}\left(\left(m_{D}^{\dagger} m_{D}\right)_{j k}\left(m_{D}^{\dagger} m_{D}\right)_{j k}\right) \frac{1}{16 \pi}\right. \\
& \left.\times\left(I\left(x_{k}\right)+\frac{\sqrt{x_{k}}}{1-x_{k}}\right)\right] \frac{1}{\left(m_{D}^{\dagger} m_{D}\right)_{j j}} \\
\simeq & \frac{g^{2}}{M_{W}^{2}} \sum_{k \neq j}\left[\left(M_{k}\right)^{2} \operatorname{Im}\left(\left(G^{\dagger} G\right)_{j k}\left(G^{\dagger} G\right)_{j k}\right) \frac{1}{16 \pi}\right. \\
& \left.\times\left(I\left(x_{k}\right)+\frac{\sqrt{x_{k}}}{1-x_{k}}\right)\right] \frac{1}{\left(G^{\dagger} G\right)_{j j}} .
\end{aligned}
$$

In Eq. (20) $M_{k}$ are the heavy neutrino masses and we have neglected terms of order $v^{2} / M_{k}^{2}$. The variable $x_{k}$ is defined 
as $x_{k}=M_{k}^{2} / M_{j}^{2}$ and the function $I\left(x_{k}\right)$ is given by $I\left(x_{k}\right)=$ $\sqrt{x_{k}}\left(1+\left(1+x_{k}\right)\left(\log x_{k}-\log \left(1+x_{k}\right)\right)\right)$. Equation (20) has been obtained after summing over all charged leptons $l_{i}^{ \pm}(i=e, \mu, \tau)$. From the Casas and Ibarra parametrization we obtain

$$
m_{D}^{\dagger} m_{D}=\sqrt{D} R^{\dagger} d R \sqrt{D} .
$$

In this framework, leptogenesis is insensitive to the low energy $C P$-violating phases appearing in $U$ and can occur even without $C P$ violation at low energies [23]. Actually, leptogenesis depends on other parameters beyond $\varepsilon_{N_{j}}$ and involves thermodynamic processes that have been analyzed by several authors [33-36]. It was pointed out recently that, under certain conditions, flavor matters in leptogenesis [37-42]. In this case we must take into account the separate lepton $i$ family asymmetry generated from the decay of the $k$ th heavy Majorana neutrino which depends on the combinations [38] $\operatorname{Im}\left(\left(m_{D}^{\dagger} m_{D}\right)_{k k^{\prime}}\left(m_{D}^{*}\right)_{i k} \times\right.$ $\left.\left(m_{D}\right)_{i k^{\prime}}\right)$ and $\operatorname{Im}\left(\left(m_{D}^{\dagger} m_{D}\right)_{k^{\prime} k}\left(m_{D}^{*}\right)_{i k}\left(m_{D}\right)_{i k^{\prime}}\right)$. Clearly, when one works with separate flavors, the matrix $U$ does not cancel out, and one is led to the interesting possibility of having viable leptogenesis even in the case of $R$ being a real matrix [43-46].

Next, we show explicitly that four-zero textures lead, in general, to $C P$ violation both at low and high energies. The strength of leptonic $C P$ violation of Dirac-type, which can be observable through neutrino oscillations, is controlled by the WB $C P$-odd invariant [47]

$$
\begin{gathered}
I_{1} \equiv \operatorname{tr}\left[h_{\mathrm{eff}}, h_{l}\right]^{3}=-6 i \Delta I_{C P}, \quad \text { with } \\
I_{C P} \equiv \operatorname{Im}\left(h_{\mathrm{eff} 12} h_{\mathrm{eff} 31} h_{\mathrm{eff} 23}\right)
\end{gathered}
$$

where $h_{\text {eff }}=m_{\text {eff }} m_{\text {eff }}^{\dagger}, h_{l}=m_{l} m_{l}^{\dagger}$, and $\Delta=\left(m_{\mu}^{2}-m_{e}^{2}\right) \times$ $\left(m_{\tau}^{2}-m_{e}^{2}\right)\left(m_{\tau}^{2}-m_{\mu}^{2}\right)$. In order to show that this $C P$-odd invariant does not vanish, in spite of the four zeros in $m_{D}$, we have to examine the structure of $h_{\text {eff }}$. For definiteness, let us consider the configuration

$$
m_{D}=\left[\begin{array}{ccc}
a_{1} & a_{2} & a_{3} \\
b_{1} & 0 & 0 \\
0 & 0 & c_{3}
\end{array}\right]
$$

belonging to case (c), category (i). Three phases can be rephased away so that one is left with only two phases, which can be placed, for instance, at the entries $(1,1)$ and $(1,2)$. From Eq. (23) and the definition of $m_{\text {eff }}$, one obtains the following structure for the latter:

$$
m_{\text {eff }} \equiv\left[\begin{array}{lll}
m_{11} & m_{12} & m_{13} \\
m_{12} & m_{22} & m_{23} \\
m_{13} & m_{23} & m_{33}
\end{array}\right]=\left[\begin{array}{ccc}
c_{11} & c_{12} & r_{13} \\
c_{12} & r_{22} & 0 \\
r_{13} & 0 & r_{33}
\end{array}\right],
$$

where entries labeled with a $c$ are complex and those labeled with an $r$ are real. With these choices there are two complex entries in $m_{\text {eff }}$. From this equation we obtain

$$
\begin{aligned}
I_{C P}= & \operatorname{Im}\left[\left|m_{13}\right|^{2}\left(m_{12}^{2} m_{22}^{*} m_{11}^{*}\right)+\left|m_{12}\right|^{2}\left(m_{11} m_{33} m_{13}^{* 2}\right)\right. \\
& \left.+m_{12}^{2} m_{13}^{* 2} m_{22}^{*} m_{33}\right] .
\end{aligned}
$$

One may note that each one of the three terms contributing to $I_{C P}$ is rephasing invariant. It is clear that $I_{C P}$ does not vanish for the $m_{\text {eff }}$ of Eq. (24).

It is well known that at low energies there are three $C P$-violating phases in $U$, one of the Dirac-type and two of the Majorana-type. The question of finding the $C P$-odd WB invariants that would be sufficient to control $C P$ violation at low energies was first addressed in Ref. [47], and more recently in Ref. [48]. In particular, it was pointed out that requiring the vanishing of the WB invariant of Eq. (22) together with the two WB invariants,

$$
\begin{gathered}
I_{2} \equiv \operatorname{Im} \operatorname{tr}\left[h_{l}\left(m_{\mathrm{eff}} m_{\mathrm{eff}}^{*}\right)\left(m_{\mathrm{eff}} h_{l}^{*} m_{\mathrm{eff}}^{*}\right)\right], \\
I_{3} \equiv \operatorname{Tr}\left[\left(m_{\mathrm{eff}}^{*} h_{l} m_{\mathrm{eff}}, h_{l}^{*}\right]^{3},\right.
\end{gathered}
$$

provides, in general, necessary and sufficient conditions for low energy $C P$ invariance [48]. The invariant of Eq. (27) was first proposed in Ref. [49], where it was shown that it has the special feature of being sensitive to Majorana-type $C P$ violation even in the limit of three exactly degenerate Majorana neutrinos. Other relevant cases can be found in Ref. [50]. The fact that, for the four-zero texture of Eq. (23), none of the three WB invariants vanishes in general, shows that this texture leads to both Dirac- and Majorana-type $C P$ violation at low energies. The same applies to the other four-zero textures.

So far, we have only considered leptonic $C P$ violation at low energies. Leptogenesis is a high energy phenomenon requiring $C P$ violation. In the unflavored case the relevant phases are those in $m_{D}^{\dagger} m_{D}$ as shown in Eq. (20). In this case one may also write a set of three independent WB invariants [22]:

$$
\begin{gathered}
I_{4} \equiv \operatorname{Im} \operatorname{Tr}\left[h_{D} H M_{R}^{*} h_{D}^{*} M_{R}\right], \\
I_{5} \equiv \operatorname{Im} \operatorname{Tr}\left[h_{D} H^{2} M_{R}^{*} h_{D}^{*} M_{R}\right], \\
I_{6} \equiv \operatorname{Im} \operatorname{Tr}\left[h_{D} H^{2} M_{R}^{*} h_{D}^{*} M_{R} H\right],
\end{gathered}
$$

where $h_{D}=m_{D}^{\dagger} m_{D}$ and $H=M_{R}^{\dagger} M_{R}$. These three would have to vanish if $C P$ were to be conserved. The condition for the vanishing of $I_{4}$ was first given in Ref. [51]. The evaluation of these invariants, in the WB with diagonal $M_{R}$, shows that, in the case of a nondegenerate $D$ and assuming no cancellations, they can all simultaneously vanish only if all imaginary parts of $\left(h_{D i j}\right)^{2}$ are absent. Now it turns out that textures of category (ii) always have one zero off-diagonal entry in $h_{D}$ due to the orthogonality of two columns of $m_{D}$, but the other two off-diagonal elements are, in general, nonzero. The same goes for those textures in category (i) that also have two orthogonal columns. The remaining textures in category (i) have, in 
general, three nonzero complex $h_{D i j}$ but their phases are constrained to be cyclic, i.e., $\operatorname{Im}\left(h_{D 12} h_{D 31} h_{D 23}\right)=0$. The fact that not all imaginary parts of $\left(h_{D i j}\right)^{2}$ vanish simultaneously in any of the four-zero textures shows that they admit $C P$ violation at high energies, relevant for leptogenesis.

\section{IMPLICATIONS FROM MODELS WITH FOUR-ZERO TEXTURES}

\section{A. Low energy physics}

Let us start by summarizing what is presently known about neutrino masses and leptonic mixing. We choose to parametrize the PMNS mixing matrix as [52]

$$
U=\left(\begin{array}{ccc}
c_{12} c_{13} & s_{12} c_{13} & s_{13} e^{-i \delta} \\
-s_{12} c_{23}-c_{12} s_{23} s_{13} e^{i \delta} & c_{12} c_{23}-s_{12} s_{23} s_{13} e^{i \delta} & s_{23} c_{13} \\
s_{12} s_{23}-c_{12} c_{23} s_{13} e^{i \delta} & -c_{12} s_{23}-s_{12} c_{23} s_{13} e^{i \delta} & c_{23} c_{13}
\end{array}\right) \cdot P
$$

where $c_{i j} \equiv \cos \theta_{i j}, s_{i j} \equiv \sin \theta_{i j}$, with all $\theta_{i j}$ in the first quadrant, $\delta$ being a Dirac-type phase and $P=$ $\operatorname{diag}\left(1, e^{i \alpha}, e^{i \beta}\right)$ with $\alpha$ and $\beta$ denoting the phases associated with the Majorana character of neutrinos.

The current experimental bounds on neutrino masses and leptonic mixing are [52]

$$
\begin{gathered}
\Delta m_{21}^{2}=8.0_{-0.3}^{+0.4} \times 10^{-5} \mathrm{eV}^{2}, \\
\sin ^{2}\left(2 \theta_{12}\right)=0.86_{-0.04}^{+0.03}, \\
\left|\Delta m_{32}^{2}\right|=(1.9 \text { to } 3.0) \times 10^{-3} \mathrm{eV}^{2}, \\
\sin ^{2}\left(2 \theta_{23}\right)>0.92, \\
\sin ^{2} \theta_{13}<0.05,
\end{gathered}
$$

with $\Delta m_{i j}^{2} \equiv m_{j}^{2}-m_{i}^{2}$. The allowed ranges for the parameters listed above correspond to an impressive degree of precision. The angle $\theta_{23}$ may be maximal (i.e., $\pi / 4$ ). In contrast, maximal mixing for $\theta_{12}$ is already ruled out experimentally. At the moment there is only an experimental upper bound on the angle $\theta_{13}$. A value of $\theta_{13}$ close to the present bound would be good news for the prospects of detecting low energy leptonic $C P$ violation, mediated through a Dirac-type phase. The strength of the latter is given by

$$
\begin{aligned}
\mathcal{J}_{C P} & \equiv \operatorname{Im}\left[U_{11} U_{22} U_{12}^{*} U_{21}^{*}\right] \\
& =\frac{1}{8} \sin \left(2 \theta_{12}\right) \sin \left(2 \theta_{13}\right) \sin \left(2 \theta_{23}\right) \cos \left(\theta_{13}\right) \sin \delta,
\end{aligned}
$$

which would in this case be of order $10^{-2}$, for $\sin \delta$ of order 1. A similar quantity defined in terms of the elements of the Cabibbo-Kobayashi-Maskawa matrix is meaningful in the quark sector [53,54], and the corresponding value is of the order of $10^{-5}$. It is not yet known whether the ordering of the light neutrino masses is normal, i.e. $m_{1}<m_{2}<m_{3}$, or inverted, i.e. $m_{3}<m_{1}<m_{2}$. The scale of the neutrino masses is not yet established. The spectrum may vary from a large hierarchy between the two lightest neutrino masses to three quasidegenerate masses. Examples of the possible extreme cases are

(a)

$$
\begin{gathered}
\left.m_{1} \sim 0 \text { (or e.g. } \sim 10^{-6} \mathrm{eV}\right), \quad m_{2} \simeq 0.009 \mathrm{eV}, \\
m_{3} \simeq 0.05 \mathrm{eV}
\end{gathered}
$$

corresponding to normal spectrum, hierarchical, or else

$$
\text { (b) } \begin{aligned}
m_{3} & \sim 0\left(\text { or e.g. } \sim 10^{-6} \mathrm{eV}\right), \\
m_{1} & \simeq m_{2} \simeq 0.05 \mathrm{eV}
\end{aligned}
$$

corresponding to inverted spectrum, hierarchical, or else

$$
\text { (c) } m_{1} \simeq 1 \mathrm{eV}, \quad m_{2} \simeq 1 \mathrm{eV}, \quad m_{3} \simeq 1 \mathrm{eV}
$$

corresponding to almost degeneracy.

As explained below, the conditions obtained in Sec. IV are not all compatible with each of these scenarios. Finally, we note that it is not yet established whether or not neutrinos are Majorana particles, and therefore, at the moment, there are no restrictions on the Majorana phases $\alpha, \beta$.

The low energy implications of patterns in category (i), which, as was already pointed out, lead to one off-diagonal set of zeros in the symmetric matrix $m_{\text {eff }}$, were studied in detail in Ref. [4]. The main conclusions in this paper are that no off-diagonal entry in $m_{\text {eff }}$ can vanish in the case of $\theta_{13}$ equal to zero. Implications of one zero in the first row of $m_{\text {eff }}$ do not differ much in the two possible such cases due to the approximate $\mu-\tau$ exchange symmetry [5557]. In this case all values of $m_{1}$ are allowed from extreme hierarchy to almost degeneracy, and so are both possible orderings of neutrino masses. For $\left(m_{\mathrm{eff}}\right)_{23}=\left(m_{\mathrm{eff}}\right)_{32}=0$ both normal hierarchy and inverted hierarchy are excluded.

Let us consider category (ii) which implies the constraints of Eq. (19). Taking into account Eq. (4) this equation can be written as

$$
\sum_{r<s} m_{r} m_{s}\left(U_{i r} U_{k s}-U_{i s} U_{k r}\right)\left(U_{i r} U_{j s}-U_{i s} U_{j r}\right)=0
$$

with $i, j, k$ different from each other and no sum implied, and the indices $r, s$ ranging from 1 to 3 .

With the explicit parametrization of Eq. (31), we obtain simple exact analytic relations for each of the classes in category (ii).

For class (ii)(a) the exact form of this constraint is 


$$
\begin{aligned}
-m_{1} m_{2} e^{2 i \alpha} c_{23} s_{23} c_{13}^{2}+m_{1} m_{3} e^{2 i \beta}[ & \left.c_{12}^{2} c_{23} s_{23}+c_{12} s_{12}\left(c_{23}^{2}-s_{23}^{2}\right) s_{13} e^{-i \delta}-s_{12}^{2} c_{23} s_{23} s_{13}^{2} e^{-2 i \delta}\right] \\
& +m_{2} m_{3} e^{2 i(\alpha+\beta)}\left[s_{12}^{2} c_{23} s_{23}+c_{12} s_{12}\left(s_{23}^{2}-c_{23}^{2}\right) s_{13} e^{-i \delta}-c_{12} c_{23} s_{23} s_{13}^{2} e^{-2 i \delta}\right]=0 .
\end{aligned}
$$

An interesting feature of this expression is the fact that all terms sensitive to Dirac-type $C P$ violation are doubly suppressed since they are multiplied either by $s_{13}^{2}$ or by the factor $\left(c_{23}^{2}-s_{23}^{2}\right) s_{13}$, and it is already known experimentally that $\theta_{13}$ corresponds to small or no mixing and $\theta_{23}$ is maximal or close to maximal. Therefore, this expression can be very well approximated by

$$
-m_{1} m_{2} e^{2 i \alpha} c_{23} s_{23} c_{13}^{2}+m_{1} m_{3} e^{2 i \beta} c_{12}^{2} c_{23} s_{23}+m_{2} m_{3} e^{2 i(\alpha+\beta)} s_{12}^{2} c_{23} s_{23}=0 .
$$

For class (ii)(b) we have the exact relation

$$
\begin{array}{r}
-m_{1} m_{2} e^{2 i \alpha} c_{23} c_{13} s_{13} e^{i \delta}+m_{1} m_{3} e^{2 i \beta}\left(c_{12} s_{12} s_{23} c_{13}+s_{12}^{2} c_{23} c_{13} s_{13} e^{-i \delta}\right) \\
+m_{2} m_{3} e^{2 i(\alpha+\beta)}\left(-c_{12} s_{12} s_{23} c_{13}+c_{12}^{2} c_{23} c_{13} s_{13} e^{-i \delta}\right)=0
\end{array}
$$

Class (ii)(c) exactly verifies

$$
\begin{array}{r}
-m_{1} m_{2} e^{2 i \alpha} s_{23} c_{13} s_{13} e^{i \delta}+m_{1} m_{3} e^{2 i \beta}\left(-c_{12} s_{12} c_{23} c_{13}+s_{12}^{2} s_{23} c_{13} s_{13} e^{-i \delta}\right) \\
+m_{2} m_{3} e^{2 i(\alpha+\beta)}\left(c_{12} s_{12} c_{23} c_{13}+c_{12}^{2} s_{23} c_{13} s_{13} e^{-i \delta}\right)=0
\end{array}
$$

This equation can be obtained from the previous one by interchanging $s_{23}$ with $c_{23}$ and by changing the sign of the terms that do not depend on the Dirac phase.

It is clear from these expressions that the main features of low energy physics coming out of these textures do not crucially depend on the possible existence of $C P$ violation. In order to get a feeling of the main features of the implications of the constraints given by Eqs. (39), (41), and (42), let us take as a first approximation the Harrison, Perkins, and Scott (HPS) mixing matrix [58]

$$
U=\left[\begin{array}{ccc}
\frac{2}{\sqrt{6}} & \frac{1}{\sqrt{3}} & 0 \\
-\frac{1}{\sqrt{6}} & \frac{1}{\sqrt{3}} & \frac{1}{\sqrt{2}} \\
-\frac{1}{\sqrt{6}} & \frac{1}{\sqrt{3}} & -\frac{1}{\sqrt{2}}
\end{array}\right],
$$

which is consistent with present experimental data, and corresponds to $\theta_{23}$ maximal, $\theta_{13}$ zero and $c_{12}=2 / \sqrt{6}$ and no $C P$ violation. Obviously, a detailed analysis would require the variation of $\theta_{13}$, as well as of $\theta_{12}$ and $\theta_{23}$, inside their allowed ranges and also to take into consideration the possibility of $C P$ violation.

Equations (39) and (43) lead to

$$
\frac{1}{2} m_{1} m_{2}-\frac{1}{3} m_{1} m_{3}-\frac{1}{6} m_{2} m_{3}=0
$$

and, as already explained, Eq. (44) corresponds to ignoring terms with a double suppression. In the $C P$-conserving limit, light neutrinos may have different $C P$ parities [59]; therefore there are several possible ways of obtaining the necessary cancellations. Normal ordering with strong hierarchy is ruled out since for $m_{1} \ll m_{2}$ there would be only one dominant term, the one in $m_{2} m_{3}$. Hierarchy in the masses implies that the magnitude of the term in $m_{2} m_{3}$ is close to $7 \times 10^{-5} \mathrm{eV}^{2}$. The strongest allowed hierarchy consistent with the above constraint favors the larger $\theta_{13}$ values; a numerical example obtained for maximal $\theta_{23}$ and the central value of $\theta_{12}$, with cancellations already of order $10^{-10} \mathrm{eV}^{2}$, is

$$
\begin{aligned}
& m_{1}=0.00333071221 \mathrm{eV}, \\
& m_{2}=-0.00954429898 \mathrm{eV}, \\
& m_{3}=0.0501108132 \mathrm{eV}, \\
& s_{13}=0.198669 .
\end{aligned}
$$

Obviously the number of significant digits in the above numerical result is meaningless from the experimental point of view and is only given to be consistent with the degree of cancellation claimed above. Here $m_{2}$ is no more than a factor of 3 higher than $m_{1}$, which corresponds to a weak normal hierarchy. As $m_{1}$ decreases, cancellations cease to occur and the difference tends to the value of the dominant term which is the term in $m_{2} m_{3}$. The situation would change for larger values of $\theta_{13}$ which are already ruled out, and likewise, for instance, for a small solar angle, $\left(\theta_{12}\right)$, already excluded, as was pointed out in [25], where a particular example of a texture of this class was considered, since in this case the term in $m_{2} m_{3}$ present in Eq. (40) would be suppressed by $s_{12}^{2}$. Strong inverse hierarchy is also ruled out since it would leave the dominant term in $m_{1} m_{2}$ without the possibility of cancellation. Quasidegeneracy can be accommodated within the present range of experimental values for the mixing angles.

Case (b) in category (ii) obeys the constraint of Eq. (41). The coefficient of $m_{1} m_{2}$ is zero for the HPS matrix, and in this case we are left with

$$
-\frac{1}{3} m_{1} m_{3}+\frac{1}{3} m_{2} m_{3}=0
$$


For nonzero $\theta_{13}$ the term in $m_{1} m_{2}$ is suppressed but cannot be discarded for $m_{3} \ll m_{1}, m_{2}$, i.e., inverse hierarchy. In the case of inverse hierarchy the necessary cancellation of the three terms may occur. Almost degeneracy can also be accommodated, provided $\theta_{13}$ is different from zero. For $\theta_{13}=0$ the coefficient of $m_{1} m_{3}$ would be exactly equal to the coefficient of $m_{2} m_{3}$, and this relation could not be verified, since it would imply $m_{1}=m_{2}$.

Case (c) in category (ii) is very similar to case (b). The resulting equation for the HPS matrix coincides with Eq. (45), and the conclusions are the same as in case (b), category (ii).

All cases in category (ii) are thus incompatible with a strong hierarchy and normal ordering, i.e. for $m_{1} \ll m_{2}$.

\section{B. Relating leptogenesis to low energy physics}

Zero textures in $m_{D}$ allow one to relate the matrix $R$, relevant to leptogenesis, to the light neutrino masses and low energy leptonic mixing. In fact, it is clear from Eq. (5) that each zero in $m_{D}$ leads to an orthogonality condition between one column of the matrix $R$ and one row of the matrix $U \sqrt{d}$ of the form

$$
\left(m_{D}\right)_{i j}=0 \Rightarrow(U)_{i k} \sqrt{d_{k k}} R_{k j}=0 .
$$

It was already pointed out [26] that the connection between leptogenesis and low energy physics could be easily established in a particular case that falls into category (ii), since in this case one can fully express the matrix $R$ in terms of light neutrino masses and low energy leptonic mixing. The same is true for all other cases in category (ii) as well as for the cases that fall into category (i) as shown below. The example given in Ref. [26] can be generalized in the following way.

In category (i) there is always in $m_{D}$ one column with two zeros and two columns with one zero each. Let $l$ be the column with two zeros and $a$ and $b$ the columns with one zero only. In this case we can write

$$
\begin{gathered}
\left(\vec{R}_{l}\right)_{i}=\left(\varepsilon_{i j k}(U)_{p j} \sqrt{m_{j}}(U)_{q k} \sqrt{m_{k}}\right) \frac{1}{N_{l}}, \\
\left(\vec{R}_{a}\right)_{i}=\left(\varepsilon_{i j k}(U)_{r j} \sqrt{m_{j}}\left(R_{l}\right)_{k}\right) \frac{1}{N_{a}}, \\
\left(\vec{R}_{b}\right)_{i}=\left(\varepsilon_{i j k}(U)_{s j} \sqrt{m_{j}}\left(R_{l}\right)_{k}\right) \frac{1}{N_{b}},
\end{gathered}
$$

where $p$ and $q$ are the rows with zeros in the column $l$. Moreover, $r$ and $s$ are the rows where the zeros are in columns $a$ and $b$, respectively. The $\vec{R}_{i}$ are the columns of the matrix $R$ and the $N_{i}$ are complex normalization factors, with phases such that $\vec{R}_{i}^{2}=1$. It is easy to show that the columns $\vec{R}_{a}$ and $\vec{R}_{b}$ are indeed orthogonal to each other by using the constraint $m_{\text {eff } r s}=0$ valid for each case in category (i).
In Ref. [25] the relation between leptogenesis and $C P$ violation at low energies in two cases falling into category (i) were analyzed in detail in the case of hierarchical heavy Majorana neutrinos and also in the case of twofold quasidegeneracy of the heavy neutrinos.

In category (ii) there is in $m_{D}$ always one column without zeros, and each of the other two columns has two zeros. Now let $l$ be the column without zeros and $a$ and $b$ the columns with two zeros. In this case we can write

$$
\begin{gathered}
\left(\vec{R}_{a}\right)_{i}=\left(\varepsilon_{i j k}(U)_{p j} \sqrt{m_{j}}(U)_{q k} \sqrt{m_{k}}\right) \frac{1}{N_{a}}, \\
\left(\vec{R}_{b}\right)_{i}=\left(\varepsilon_{i j k}(U)_{r j} \sqrt{m_{j}}(U)_{s k} \sqrt{m_{k}}\right) \frac{1}{N_{b}}, \\
\left(\vec{R}_{l}\right)_{i}=\varepsilon_{i j k}\left(\vec{R}_{a}\right)_{j}\left(\vec{R}_{b}\right)_{k} .
\end{gathered}
$$

Here $p$ and $q$ are the rows with zeros in column $a$. Furthermore, $r$ and $s$ are the rows with zeros in column $b$, while $N_{i}$ denote normalization factors. It is easy to show that $\vec{R}_{a}$ and $\vec{R}_{b}$ are indeed orthogonal to each other for each case in category (ii) by using Eq. (19).

All four-zero textures analyzed in this paper allow one to completely specify the matrix $R$, in terms of light neutrino masses and the elements of the PMNS matrix. It is clear that $R$ can only be complex if there is $C P$ violation at low energies.

\section{SUMMARY AND CONCLUSIONS}

We have made a systematic study of all allowed fourzero textures in the neutrino Dirac mass matrix $m_{D}$, in the framework of the type I seesaw mechanism, without vanishing neutrino masses. In order for this study to be meaningful, one has to choose a specific WB. Without loss of generality, we have chosen to work in the WB where the charged lepton and the right-handed neutrino mass matrices are both diagonal, real. Assuming that no neutrino mass vanishes and taking into account the experimental evidence that no leptonic family decouples from the other two, we have shown that four is the maximal number of zeros allowed in $m_{D}$. We have found the following remarkable result: the allowed four-zero textures in the neutrino Yukawa coupling matrices automatically lead to one of two patterns in $m_{\nu}$. Either the latter has only one pair of symmetric off-diagonal zeros, or else it has no zero element but a vanishing subdeterminant condition. In the derivation of this result, we have implicitly assumed the absence of any fine-tuning between the parameters of $m_{D}$ and those of $M_{R}$ which would lead to special cancellations.

Our analysis also applies to scenarios where, instead of zeros, one has a set of extremely suppressed entries [60], as one often encounters in the Froggatt-Nielsen approach. Of course, renormalization group effects, especially for qua- 
sidegenerate and inverted hierarchical neutrinos [12], will change at least some of the zeros in $m_{D}$ into small entries.

We have also explored the phenomenological consequences of the above-mentioned textures. In particular, we have shown that they lead to a close connection between leptogenesis and low energy measurables such as neutrino masses and mixing angles. The establishment of such a connection in the leptonic sector between physics at low and very high energies is an important goal and provides an additional motivation for considering texture zeros in the leptonic sector.

\section{ACKNOWLEDGMENTS}

This work was partially supported by Fundação para a Ciência e a Tecnologia (FCT, Portugal) through Project
No. POCTI/FNU/44409/2002, No. PDCT/FP/63914/2005, No. PDCT/FP/63912/2005, and No. CFTP-FCT UNIT 777, which are partially funded through POCTI (FEDER). The work of D.E. C. is supported by CFTPFCT UNIT 777. The work of G. C. B. was supported by the Alexander von Humboldt Foundation. The work of P. R. has been supported in part by DAE (BRNS). G. C. B. would like to thank Andrzej J. Buras for the kind hospitality at TUM. P. R. acknowledges the generous hospitality of CFTP (IST), Lisboa, where this investigation started. G.C. B. and M. N.R. are grateful for the warm hospitality of the CERN Physics Department (PH) Theory Division $(\mathrm{TH})$, where the work was finalized.
[1] P.H. Frampton, S. L. Glashow, and D. Marfatia, Phys. Lett. B 536, 79 (2002).

[2] G. C. Branco, R. Gonzalez Felipe, F. R. Joaquim, and T. Yanagida, Phys. Lett. B 562, 265 (2003).

[3] S. Dev, S. Kumar, S. Verma, and S. Gupta, Phys. Rev. D 76, 013002 (2007).

[4] A. Merle and W. Rodejohann, Phys. Rev. D 73, 073012 (2006).

[5] W. Grimus, A. S. Joshipura, L. Lavoura, and M. Tanimoto, Eur. Phys. J. C 36, 227 (2004)

[6] S. Kaneko, H. Sawanaka, T. Shingai, M. Tanimoto, and K. Yoshioka, arXiv:hep-ph/0703250.

[7] C. D. Froggatt and H. B. Nielsen, Nucl. Phys. B147, 277 (1979).

[8] C. Hagedorn, J. Kersten, and M. Lindner, Phys. Lett. B 597, 63 (2004).

[9] S. Antusch, J. Kersten, M. Lindner, M. Ratz, and M. A. Schmidt, J. High Energy Phys. 03 (2005) 024.

[10] J. W. Mei, Phys. Rev. D 71, 073012 (2005).

[11] A. Dighe, S. Goswami, and P. Roy, Phys. Rev. D 73, 071301 (2006).

[12] A. Dighe, S. Goswami, and P. Roy, Phys. Rev. D 76, 096005 (2007).

[13] A. Ibarra and G. G. Ross, Phys. Lett. B 591, 285 (2004).

[14] W. 1. Guo, Z. z. Xing, and S. Zhou, Int. J. Mod. Phys. E 16, 1 (2007).

[15] M. Fukugita and T. Yanagida, Phys. Lett. B 174, 45 (1986).

[16] M. B. Gavela, P. Hernandez, J. Orloff, O. Pene, and C. Quimbay, Nucl. Phys. B430, 382 (1994).

[17] P. Huet and E. Sather, Phys. Rev. D 51, 379 (1995).

[18] G. W. Anderson and L. J. Hall, Phys. Rev. D 45, 2685 (1992).

[19] W. Buchmuller, Z. Fodor, T. Helbig, and D. Walliser, Ann. Phys. (N.Y.) 234, 260 (1994).

[20] K. Kajantie, M. Laine, K. Rummukainen, and M.E. Shaposhnikov, Nucl. Phys. B466, 189 (1996).
[21] L. Fromme, S. J. Huber, and M. Seniuch, J. High Energy Phys. 11 (2006) 038.

[22] G. C. Branco, T. Morozumi, B. M. Nobre, and M. N. Rebelo, Nucl. Phys. B617, 475 (2001)

[23] M. N. Rebelo, Phys. Rev. D 67, 013008 (2003).

[24] S. Kaneko and M. Tanimoto, Phys. Lett. B 551, 127 (2003).

[25] G. C. Branco, R. Gonzalez Felipe, F. R. Joaquim, I. Masina, M. N. Rebelo, and C. A. Savoy, Phys. Rev. D 67, 073025 (2003).

[26] G. C. Branco, M. N. Rebelo, and J. I. Silva-Marcos, Phys. Lett. B 633, 345 (2006).

[27] B. Pontecorvo, Zh. Eksp. Teor. Fiz. 34, 247 (1957) [Sov. Phys. JETP 7, 172 (1958)];Z. Maki, M. Nakagawa, and S. Sakata, Prog. Theor. Phys. 28, 870 (1962); B. Pontecorvo, Zh. Eksp. Teor. Fiz. 53, 1717 (1967) [Sov. Phys. JETP 26, 984 (1968)].

[28] J. A. Casas and A. Ibarra, Nucl. Phys. B618, 171 (2001).

[29] A. Kageyama, S. Kaneko, N. Shimoyama, and M. Tanimoto, Phys. Lett. B 538, 96 (2002).

[30] G. C. Branco, D. Emmanuel-Costa, R. G. Felipe, and H. Serodio, arXiv:0711.1613.

[31] G. C. Branco and L. Lavoura, Nucl. Phys. B278, 738 (1986).

[32] J. Liu and G. Segre, Phys. Rev. D 48, 4609 (1993); M. Flanz, E. A. Paschos, and U. Sarkar, Phys. Lett. B 345, 248 (1995); 382, 447(E) (1996); L. Covi, E. Roulet, and F. Vissani, Phys. Lett. B 384, 169 (1996); A. Pilaftsis, Phys. Rev. D 56, 5431 (1997); W. Buchmüller and M. Plümacher, Phys. Lett. B 431, 354 (1998).

[33] W. Buchmuller, P. Di Bari, and M. Plumacher, Nucl. Phys. B665, 445 (2003).

[34] W. Buchmuller, P. Di Bari, and M. Plumacher, Ann. Phys. (N.Y.) 315, 305 (2005).

[35] W. Buchmuller, P. Di Bari, and M. Plumacher, New J. Phys. 6, 105 (2004).

[36] G. F. Giudice, A. Notari, M. Raidal, A. Riotto, and A. Strumia, Nucl. Phys. B685, 89 (2004). 
[37] R. Barbieri, P. Creminelli, A. Strumia, and N. Tetradis, Nucl. Phys. B575, 61 (2000).

[38] T. Fujihara, S. Kaneko, S. Kang, D. Kimura, T. Morozumi, and M. Tanimoto, Phys. Rev. D 72, 016006 (2005).

[39] A. Pilaftsis and T.E. J. Underwood, Phys. Rev. D 72, 113001 (2005).

[40] A. Abada, S. Davidson, F. X. Josse-Michaux, M. Losada, and A. Riotto, J. Cosmol. Astropart. Phys. 04 (2006) 004.

[41] E. Nardi, Y. Nir, E. Roulet, and J. Racker, J. High Energy Phys. 01 (2006) 164.

[42] A. Abada, S. Davidson, A. Ibarra, F. X. Josse-Michaux, M. Losada, and A. Riotto, J. High Energy Phys. 09 (2006) 010.

[43] G. C. Branco, A.J. Buras, S. Jager, S. Uhlig, and A. Weiler, J. High Energy Phys. 09 (2007) 004.

[44] S. Pascoli, S. T. Petcov, and A. Riotto, Phys. Rev. D 75, 083511 (2007).

[45] G.C. Branco, R. Gonzalez Felipe, and F. R. Joaquim, Phys. Lett. B 645, 432 (2007).

[46] S. Uhlig, J. High Energy Phys. 11 (2007) 066.

[47] G. C. Branco, L. Lavoura, and M. N. Rebelo, Phys. Lett. B 180, 264 (1986).
[48] H. K. Dreiner, J. S. Kim, O. Lebedev, and M. Thormeier, Phys. Rev. D 76, 015006 (2007).

[49] G. C. Branco, M. N. Rebelo, and J. I. Silva-Marcos, Phys. Rev. Lett. 82, 683 (1999).

[50] G. C. Branco and M.N. Rebelo, New J. Phys. 7, 86 (2005).

[51] A. Pilaftsis, Phys. Rev. D 56, 5431 (1997).

[52] W. M. Yao et al. (Particle Data Group), J. Phys. G 33, 1 (2006).

[53] C. Jarlskog, Phys. Rev. Lett. 55, 1039 (1985).

[54] J. Bernabeu, G. C. Branco, and M. Gronau, Phys. Lett. 169B, 243 (1986).

[55] C. S. Lam, Phys. Lett. B 507, 214 (2001).

[56] W. Grimus and L. Lavoura, J. High Energy Phys. 07 (2001) 045.

[57] E. Ma, Phys. Rev. D 66, 117301 (2002).

[58] P. F. Harrison, D. H. Perkins, and W. G. Scott, Phys. Lett. B 530, 167 (2002).

[59] L. Wolfenstein, Phys. Lett. 107B, 77 (1981).

[60] F. Plentinger, G. Seidl, and W. Winter, Phys. Rev. D 76, 113003 (2007). 